\title{
Il servizio alla verità nel processo matrimoniale
}

\author{
Serving the Truth in the Marriage Process
}

Paolo BIANCHI

Vicario Giudiziale

Tribunale diocesano dell'Arcidiocesi di Milano

paoloterl@tiscali.it

Resumen: La verdad que se intenta servir en el proceso matrimonial canónico se puede entender en diferentes sentidos: como verdad de hecho, es decir, que corresponde a la real sucesión de hechos puestos a examen judicial; como verdad doctrinal que debe ser respetada en la decisión; como verdad del contenido jurídico, que enmarca la historia matrimonial en el contexto de la disciplina canónica; y, en último lugar, como verdad orientada a la salvación de las almas.

El tema de la búsqueda de la verdad es una presencia constante en las alocuciones de los Papas a la Rota Romana, desde Pío XII a Francisco, y retorna en modo claro también en el mp Mitis ludex Dominus lesus: la búsqueda y el servicio a la verdad caracterizan también esta revisión normativa.

Algunos institutos realizan la orientación del proceso hacia la verdad: la correcta comprensión del contradictorio; la superordinación del favor veritatis tanto al favor personae como al favor matrimonii; la centralidad de la certeza moral en el sistema procesal canónico; el reconocimiento del valor probatorio de las declaraciones de las partes, utilizadas en modo equilibrado y prudente.

Palabras clave: Verdad, Proceso matrimonial canónico, Certeza moral.

IUS CANONICUM / VOL. 57 / 2017 / [1-22] 83-104 ISSN 0021-325X

DOI 10.15581/016.113.007
Abstract: Truth in the canonical marriage process avails of different meanings: factual truth, the real course of the marriage events presented for judicial examination; the doctrinal truth to be respected in the decision; truth with a legal dimension, i.e. which contextualizes the marriage in terms of the canonical discipline; and finally, as truth oriented towards spiritual salvation.

The pursuit of truth is a recurring theme papal addresses to the Roman Rota, from Pius XII to Francis, and it is set out again in the Mitis ludex Dominus lesus: pursuit and service to the truth are key features of this latest revision of the regulation. Some institutions set out to guide this process towards truth: the appropriate understanding of contradictory positions; the primacy of favor veritatis in relation to both favor personae and favor matrimonii; the fundamental importance of moral certainty in the canonical process system; the recognition of the proof-value of the statements made by the parties involved, deferred to in a prudent and balanced way.

Keywords: Truth, Canonical Marriage Process, Moral Certainty. 
Q uello assegnatomi è un tema facile e difficile allo stesso tempo. Infatti, da un lato sarebbe sorprendente trovare qualcuno che sostenesse (almeno espressamente) che nelle cause di nullità matrimoniale non si debba cercare la verità, cosa che porterebbe a pensare che ogni discorso in merito sia superfluo; dall'altro ci si potrebbe domandare se sia sempre davvero così, cosa che rende invece urgente occuparsi della questione.

Ancora: da un lato, ci si potrebbe domandare di quale verità si tratti e se tale verità esista o, almeno, se sia davvero attingibile, tenendo conto di quell'orientamento piuttosto scettico che caratterizza il pensiero post moderno; dall'altro ci si potrebbe chiedere se non sia giunto il momento di superare sia un radicale pessimismo gnoseologico in favore di un maggiore (per quanto non ingenuo ma critico) realismo; sia un pessimismo della volontà, un pessimismo etico che ritiene l'uomo -pur senza nasconderne le debolezze e le incoerenze-strutturalmente incapace di tendere verso il bene, per quanto in ipotesi conosciuto.

Così, sotto il profilo del metodo, la questione dell'esistenza della verità potrebbe essere indagata secondo diverse metodologie o discipline (filosofia, psicologia, sociologia...), peraltro ultimamente piuttosto allergiche a ogni pretesa veritativa, in nome della tolleranza e della insindacabilità delle scelte individuali, nonché della connessione -data come presupposta senza vera dimostrazione- fra indifferentismo verso la verità e pace sociale.

Infine, anche la comunicazione dei risultati di ogni tipo di riflessione potrebbe essere fatta in molti modi, anche solo ad esempio relativamente alla consistenza dell'apparato critico del proprio contributo.

Per la redazione di questo contributo ho alla fine pensato di lasciarmi guidare dal titolo assegnato, che presuppone che una verità esista (anche se andrà bene precisato di che tipo di verità si tratti in questa sede); che essa vada servita, ossia riconosciuta e promossa; e, infine, che tale riconoscimento debba avvenire precisamente all'interno dello strumento processuale, anzi di quello specifico processo che è quello di dichiarazione della nullità matrimoniale.

Quanto al metodo, ho cercato di raccogliere soprattutto delle idee, limitando al massimo l'appesantimento del testo con citazioni (su un argomento del genere moltiplicabili a dismisura $\left.{ }^{1}\right)$, limitandomi tendenzialmente a quelle magisteriali.

${ }^{1}$ Devo però dichiarare -anche per un senso di riconoscenza nei loro confronti- che fra gli autori cui mi sono maggiormente ispirato nella redazione di queste note vi sono Manuel Jesús Arroba Conde, Massimo del Pozzo, Joaquín Llobell, Carlos Morán Bustos. 


\section{QUALE VERITÀ SI PRESUPPONE COME DA SERVIRE?}

1.1. Anzitutto una verità fattuale, basata sulla convinzione della possibilità di ricostruire una verità storica.

Non si può certo negare anche una dimensione soggettiva nella percezione della verità dei fatti e del loro significato, dal momento che la «verità cercata nei processi di nullità matrimoniale non è [...] una verità astratta, avulsa dal bene delle persone. È una verità che si integra nell'itinerario umano e cristiano di ogni fedele» ${ }^{2}$. Tuttavia, nemmeno si può disconoscere che vi sia un nucleo oggettivo di fatti che può essere riconosciuto, che deve essere accettato come dato storicamente accertato e sulla base del quale vedere basata la valutazione della propria condizione personale.

In questo senso si può affermare che si tratta di verità non solo in senso etico (ossia la sincerità, la lealtà di condotta nella dinamica processuale), bensì anche in senso ontologico, ossia corrispondente all'effettivo essere delle cose, per quanto qui limitatamente al loro essere enti di fatto, non di ragione.

\subsection{Una verità non solo fattuale, ma anche dottrinale.}

Nella sua allocuzione alla Rota Romana del 22 gennaio 2011, Benedetto XVI ha offerto delle considerazioni sulla omogeneità di linguaggio (nel senso del contenuto, beninteso, non solo in senso nominalistico, ossia delle parole usate) che deve sussistere fra dottrina (la visione antropologica dell'uomo e del matrimonio anche come trasfusa nell'ordinamento canonico), pastorale e prassi giudiziale: «Tutto ciò richiede [...] che l'operato dei tribunali ecclesiastici trasmetta un messaggio univoco circa ciò che è essenziale nel matrimonio, in sintonia con il Magistero e la legge canonica, parlando ad una sola voce» ${ }^{3}$.

Il processo matrimoniale canonico si struttura infatti sulla base di un diritto sostanziale che media una visione integrale della persona (e quindi del matrimonio). Ciò ad esempio ${ }^{4}$ coltivando una visione realistica (non elitaria ed idealizzata) della capacità al matrimonio (cfr. can. 1095) e tutelando la libertà personale da indebite coazioni esterne (cfr. can. 1103): distinguendo però le vere mancanze di libertà (interna ed esterna) dalle strutturali limitatezze e im-

2 Così si esprimeva BenedetTo XVI nella sua allocuzione alla Rota Romana del 28 gennaio 2006, AAS 98 (2006) 138.

3 AAS 103 (2011) 112.

${ }^{4} \mathrm{Ci}$ si riferisce qui ai difetti e vizi del consenso, che sono ormai la maxima pars dei motivi di nullità proposti nei tribunali della Chiesa, trascurando i difetti di forma e gli impedimenti. 
perfezioni della libertà umana. L'ordinamento coltiva poi il principio della responsabilità personale, ad esempio espresso dalla presunzione di cui al can. 1101 $\$ 1$, superabile solo di fronte alla prova certa di una volontà contraria (cfr. can. $1101 \$ 2)$ o condizionata in modo determinante da un errore di diritto (cfr. cann. $1096 \$ 1$ e 1099). Così come coltiva la trascendenza della persona rispetto alle sue caratteristiche accidentali, disegnando una disciplina piuttosto rigorosa in materia di errore di fatto (cfr. cann. 1097-1098). Ancora, l'ordinamento canonico promuove una concezione forte sia della libertà, che si realizza attraverso la donazione di sé tramite la formalità dell'assunzione degli impegni coniugali (cfr. can. $1057 \$ 2$ ); sia dell'amore coniugale: non già ridotto alla inclinazione sentimentale ed erotica, bensì elevato al suo livello di maggior pregio umano e morale come amore di benevolenza, che si fa carico della condivisione di un comune progetto esistenziale (il consortium vitae di cui al can. $1055 \$ 1$ ). Amore che è unico, irrevocabile e fedele (cfr. can. 1056), centrato non individualisticamente su se stesso nella smaniosa ricerca di una (insaziabile) autorealizzazione, ma aperto agli altri: al coniuge in primo luogo e ai figli.

In questi termini, il processo di nullità matrimoniale mostra -anche già sotto lo specifico profilo del diritto sostanziale- la propria corrispondenza a una considerazione integrale della persona, orientata al dono di sé nell'autotrascendimento in vista dei valori evangelici e nel superamento di una concezione individualistica ed utilitaristica della esistenza. Tale corrispondenza offre sufficienti garanzie che anche la verità dottrinale del sacramento (e comunque della concezione ecclesiale del matrimonio) sia adeguatamente servita.

Fare riferimento a una verità dottrinale da riconoscere, significa però anche rendersi conto che la responsabilità che incombe in chi la deve servire è non solo quella di non tradire la dottrina nella decisione del singolo caso, ma anche quella di considerare il valore educativo della giurisprudenza, nel testimoniare e nel trasmettere credibilmente la concezione ecclesiale del matrimonio. In questo senso, una prassi giurisdizionale per così dire destrutturante il matrimonio -ad esempio quantitativamente massiva nella applicazione dei motivi di nullità (quasi siano la regola, non un'eccezione), oppure eccessivamente creativa nell'interpretazione del loro ambito semantico (si vedano alcune estensioni del significato giuridicamente rilevante del bene dei coniugi)potrebbe nella sua proiezione futura risultare anche diseducativa rispetto alla stabilità del matrimonio cristiano, offrendo alla fin fine una contro testimonianza rispetto alle pur riaffermate caratteristiche della concezione ecclesiale del matrimonio, soprattutto la sua indissolubilità. 
Non bisogna a tale proposto dimenticare la affermazione del Papa che in Amoris laetitia ha addirittura definito la pastorale della famiglia come una «pastorale del vincolo» (n. 211), così come ha definito la spiritualità matrimoniale come «una spiritualità del vincolo abitato dall'amore divino» (n. 315).

1.3. Una verità anche giuridica, ossia corrispondente a una qualificazione giuridica dei dati di fatto; una operazione che si pone su un piano ulteriore rispetto a quello della loro materiale individuazione e che richiede una perizia di carattere tecnico.

Fare riferimento a questa dimensione non vuol dire necessariamente porsi su un piano inaccessibile alla comprensione dei fedeli o lontano dalla loro realtà esistenziale. Significa invece -anche avendo cura il più possibile di rendere comprensibili la procedura e le decisioni dei tribunali (perché gli eccessi di astrattezza sono pure possibili)- aiutare a collocare e a leggere le singole esperienze individuali nel quadro complessivo della comprensione cristiana della persona umana e del matrimonio, così come tutelate dall'ordinamento canonico.

Una separazione radicale fra realtà (esperienza esistenziale) ed idea (i valori protetti dall'ordinamento) appare poco sostenibile: a meno che si voglia teorizzare che le regole non siano altro che le concrete esperienze esistenziali. Il che, peraltro, sarebbe a sua volta una idea, una affermazione teorica, un'opzione ideologica.

1.4. Infine, una verità orientata alla salus animarum.

È quasi divenuta rituale l'invocazione di questa finalizzazione della legge canonica, spesso però ripetuta senza sue ulteriori illustrazioni e in contesti dove le conseguenze pratiche tratte da tale affermazione non appaiono sempre univoche e coerenti.

In questa sede, penso occorra ribadire il nesso intrinseco, strutturale, fra salvezza delle anime e verità. Infatti, solo sulla base del riconoscimento della verità della propria situazione esistenziale (non sulla base di un infingimento o di prospettazioni ambigue in merito) può essere costruito un sensato cammino pastorale e spirituale.

Penso utile, a tale proposito, riprendere una fortunatissima immagine di papa Francesco sulla Chiesa come ospedale da campo, utilizzata anche al n. 291 di Amoris laetitia. Tale bellissima immagine, se coerentemente svilup- 
pata, suppone di dover ammettere che non tutte le malattie o ferite siano uguali e che occorra fare di esse una diagnosi appropriata. Un errore diagnostico (ad esempio considerando tutte le malattie uguali o non riconoscendone la oggettiva gravità) non può che avere delle conseguenze negative sulla scelta e sull'efficacia della terapia. Così come il ritenere che ogni malattia sia guaribile: l'esperienza clinica (per rimanere all'interno dell'analogia che stiamo sviluppando) mostra invece che alcune malattie sono inguaribili; mentre la cura adeguata che pure si deve assicurare al paziente comporta di non illuderlo circa la natura del suo male.

Penso infine importante avere una concezione che vorrei definire escatologica della salus animarum, ossia non appiattita sulla sola dimensione storica, contingente. Infatti, solo nella condizione escatologica la salus animarum troverà il suo pieno compimento, si supererà il limite intrinseco della condizione umana, nonché troveranno piena armonia giustizia e misericordia. Occorre guardarsi da una concezione per così dire superomistica e nietzschiana dell'attività pastorale: rispettivamente nel senso o dell'onnipotenza della istituzione o della pretesa che ogni aspirazione e desiderio debbano trovare adempimento, anche giuridico. $\grave{E}$ il grande inganno o equivoco della cultura corrente, almeno nei Paesi occidentali, che porta a confondere pretesa soggettiva e diritto.

Da queste considerazioni devono discendere una profonda umiltà e un grande realismo: riconoscendo sì la necessità ma anche il limite dell'azione ecclesiale, che non può sanare o tanto meno sistemare (come spesso si dice in una prospettiva e con un linguaggio piattamente pragmatici) ogni situazione, arrestandosi di fronte al mistero di Dio e della libertà personale, la quale può anche aver commesso errori ormai irreparabili almeno nella sola prospettiva storica.

\section{IL RICONOSCIMENTO DELLA VERITÀ}

2.1. La persona umana ha il diritto e il dovere di cercare la verità e anche di adeguarsi ad essa una volta che la verità sia conosciuta, soprattutto laddove siano implicati anche risvolti di carattere religioso. Lo afferma chiaramente il testo del can. $748 \$ 1$ :

«Omnes homines veritatem in iis, quae Deum eiusque Ecclesiam respiciunt, quaerere tenentur eamque cognitam amplectendi ac servandi obligatione vi legis divinae adstringuntur et iure gaudent». 
Tale principio generale vale anche per l'argomento del quale ci occupiamo in questa sede. E, infatti, la ricerca della verità è il dovere di tutti coloro che partecipano al processo.

Questa impellente doverosità viene espressa in modo molto chiaro da quella che viene denominata concezione istituzionale del processo; una espressione volta appunto ad evidenziare che tutti coloro che vi partecipano debbono collaborare alla ricerca della verità e che ha trovato nella allocuzione alla Rota Romana di Pio XII per l'anno $1944^{5}$ la sua delineazione più compiuta. Infatti, in essa il servizio alla verità -ossia la disponibilità alla sua ricerca e al riconoscerne la forza vincolante- viene ripetutamente proposto come il fine ultimo del processo canonico e declinato puntualmente per tutte le figure che ne sono partecipi: giudice, difensore del vincolo, promotore di giustizia, avvocati, parti, testimoni e periti. Le loro funzioni sono paragonate alle membra di un corpo, che cooperano armoniosamente al conseguimento dello scopo finale dell'organismo del quale sono parte: ossia la salvezza delle anime che passa attraverso il riconoscimento della verità.

2.2. Non è comunque difficile constatare come quello della centralità della verità nel processo canonico sia un tema costante nelle allocuzioni dei Papi alla Rota Romana.

Lo stesso Pio XII affermava, nella sua allocuzione alla Rota Romana del 2 ottobre 1940, che «figlia della verità vuol essere la giustizia, se ha da farsi madre di pace» ${ }^{6}$; mentre nella celebre allocuzione del $1^{\circ}$ ottobre 1942 -nella quale viene definito il concetto di certezza morale, così centrale per il diritto processuale canonico- si afferma che «la verità è la legge della giustizia»?

Da parte sua, anche san Giovanni XXIII collegava strettamente l'essere ministerium veritatis dell'attività giudiziaria con la sua finalizzazione alla salus animarum, vista nella «prospettiva di una significazione che va oltre il tempo e le

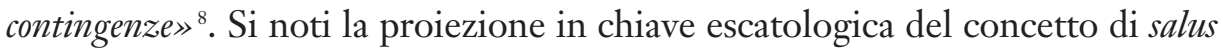
animarum della quale si diceva più sopra.

${ }^{5}$ Cfr. AAS 36 (1944) 281-290.

6 Tale allocuzione non è pubblicata in AAS, ma è reperibile in G. ERLEBACH (a cura di), Le allocuzioni dei Sommi Pontefici alla Rota Romana, Libreria Editrice Vaticana, Città del Vaticano 2004, 24, n. 12.

${ }^{7}$ AAS $34(1942) 342$.

${ }^{8}$ Così nella allocuzione del 13 dicembre 1961, AAS 53 (1961) 819. 
Anche il Beato Paolo VI, nell'allocuzione alla Rota Romana del 28 gennaio 1978, insegnava: «per garantire ai giudici l'atmosfera necessaria per un esame sereno, attento, meditato, completo ed esauriente delle questioni, per assicurare alle parti la reale possibilità di illustrare le proprie ragioni, la legge canonica prevede un cammino segnato da norme precise -il "processo" appunto-, che è come un binario di scorrimento, il cui asse è precisamente la ricerca della verità oggettiva ed il cui punto terminale è la retta amministrazione della giustizia»?

Da parte sua, san Giovanni Paolo II, esordiva nella sua prima allocuzione alla Rota Romana, il 17 febbraio 1979, indicando «la funzione giudiziaria della Chiesa al servizio della verità e della carità per l'edificazione del Corpo di Cristo» ${ }^{10}$; mentre tutta l'allocuzione del 4 febbraio 1980 è incentrata sul tema della verità come punto di forza per il raggiungimento della giustizia e della pace ${ }^{11}$, giungendo anzi ad affermare -citando teologi, canonisti e suoi predecessori- che «la giustizia ba un suo rapporto di dipendenza nei confronti della verità» per cui anche nei processi matrimoniali canonici «la verità deve essere sempre, dall'inizio fino alla sentenza, fondamento, madre e legge della giustizia». Per conseguenza: «Tutti gli atti del giudizio ecclesiastico [...] possono e debbono essere fonte di verità», soprattutto gli atti istruttori, che mirano alla ricostruzione dei fatti.

Così, nella allocuzione del 24 gennaio 1981, il Papa segnalava che sarebbe contraria alla tutela della famiglia un'attività giudiziaria che non fosse basata sulla conoscenza e sul rispetto della verità oggettiva: «L'azione giudiziaria dei tribunali ecclesiastici matrimoniali [...] dovrà aiutare la persona umana nella ricerca della verità oggettiva e quindi ad affermare questa verità, affinché la stessa persona possa essere in grado di conoscere, vivere e realizzare il progetto d'amore che Dio le ha assegnato» ${ }^{12}$.

Anche il non farsi condizionare da premesse antropologiche incompatibili con la visione cristiana dell'uomo e del matrimonio è ritenuto da Giovanni Paolo II appartenere al «ministero di verità e di carità» che i giudici esercitano nella Chiesa e per la Chiesa ${ }^{13}$, cosa che viene ripetuta l'anno successivo a proposito del servizio del difensore del vincolo, che non deve mancare di operare per «la effettiva ricerca della verità, la quale deve essere sempre "fondamento madre e legge della giustizia"» ${ }^{14}$.

\footnotetext{
AAS 70 (1978) 182.

${ }^{10}$ AAS $71(1979) 422$.

${ }_{11}$ Cfr. AAS 72 (1980) 172-178. Le citazioni subito successive, alle pp. 173 e 174.

12 AAS 73 (1981) 233-234.

13 Cfr. l'allocuzione del 5 febbraio 1987, AAS 79 (1987) 1458.

${ }^{14}$ AAS $80(1988) 1135$.
} 
Nella allocuzione alla Rota del 18 gennaio $19900^{15}$, Giovanni Paolo II sostiene poi che una sentenza di nullità matrimoniale non basata sulla verità non sarebbe che un inganno per i fedeli e che nessuna giustificazione essa potrebbe trovare in riferimento a pretesi atteggiamenti di pastoralità e di misericordia; così come lo sarebbe una interpretazione della legge che, per salvaguardare asserite esigenze delle parti, ne snaturasse le caratteristiche: «Piegare la legge canonica al capriccio o all'inventiva interpretativa, in nome di un "principio umanitario" ambiguo ed indefinito, significherebbe mortificare, prima ancora della norma, la stessa dignità dell'uomo» ${ }^{16}$.

Tutta l'allocuzione alla Rota del 28 gennaio 1994 è dedicata poi da Giovanni Paolo II al «suggestivo rapporto che intercorre tra lo splendore della verità e quello della giustizia» ${ }^{17}$, giustizia che non può essere piegata «al servizio di interessi individuali e di forme pastorali, sincere forse, ma non basate sulla verità» ${ }^{18}$. Anzi la disponibilità alla verità dovrà essere invocata come dono da tutti i partecipanti al processo ${ }^{19}$ e la verità stessa andrà accettata anche quando scomoda ed esigente ${ }^{20}$.

Sempre il Santo Pontefice nella allocuzione alla Rota Romana del 17 gennaio 1998, parla del ministero del giudice come sottomesso a «imprescindibili esigenze di verità e di giustizia» ${ }^{21}$, mentre in quella del 21 gennaio 1999, rivolge agli uditori rotali l'esortazione «a dare prevalenza, nella soluzione dei casi, alla ricerca della verità» ${ }^{22}$.

Nella allocuzione alla Rota del 28 gennaio 2002 -dedicata al tema della indissolubilità del matrimonio, sul quale Giovanni Paolo II è tornato spesso nei suoi ultimi discorsi al Tribunale Apostolico- si afferma, a proposito dei processi per l'accertamento della eventuale invalidità del patto nuziale: «Gli stessi coniugi devono essere i primi a comprendere che solo nella leale ricerca della verità si trova il loro vero bene»» ${ }^{23}$, perché una sentenza contraria alla verità non sarebbe che una ingiustizia. In questa linea, nella allocuzione del 29 gennaio 2004, il Papa affermava: «Tale processo è essenzialmente inconcepibile al di fuori

\footnotetext{
5 Cfr. AAS 82 (1990) 875.

${ }^{16}$ Così l'allocuzione alla Rota del 29 gennaio 1993, AAS 85 (1993) 1259.

7 AAS 86 (1994) 948.

${ }^{18}$ Ibid., 949.

${ }^{19}$ Cfr. ibid., 949-950.

${ }^{20}$ Cfr. ibid., 950-951.

${ }^{21}$ AAS 90 (1998) 784.

${ }^{22}$ AAS 91 (1999) 622.

23 AAS 94 (2002) 344.
} 
dell'orizzonte dell'accertamento della verità. Questo riferimento teleologico alla verità è ciò che accomuna tutti i protagonisti del processo, nonostante la diversità dei loro ruoli. [...] La tendenza ad ampliare strumentalmente le nullità, dimenticando l'orizzonte della verità oggettiva, comporta una distorsione strutturale dell'intero processo» ${ }^{24}$.

Infine, tutta l'ultima allocuzione alla Rota Romana di san Giovanni Paolo II venne dedicata alla «dimensione morale dell'attività degli operatori giuridici presso $i$ tribunali ecclesiastici, soprattutto per quel che riguarda il dovere di adeguarsi alla verità sul matrimonio» ${ }^{25}$, dovere che riguarda naturalmente anche la verità di fatto, come viene illustrato con accenti preoccupati nel prosieguo del discorso, che insiste sullo «essenziale rapporto che il processo ba con la ricerca della verità oggettiva» ${ }^{26}$.

Fin dalla sua prima allocuzione alla Rota Romana, Benedetto XVI ha trattato dell'amore per la verità come del «fondamentale punto di incontro tra diritto e pastorale» per cui il processo canonico per il riconoscimento della eventuale nullità di un matrimonio ha un «valore pastorale, che non può essere separato dall'amore alla verità» ${ }^{27}$.

Nella sua seconda allocuzione, Benedetto XVI si è particolarmente concentrato sulla verità dottrinale in merito al matrimonio «in un contesto culturale segnato dal relativismo e dal positivismo giuridico ${ }^{28}$, ribadendo la necessità di interpretazione delle norme canoniche in un contesto di ermeneutica della continuità in relazione alla tradizione ecclesiale.

Nella allocuzione del 29 gennaio 2010, Benedetto XVI ha sottoposto ad accurata analisi il rapporto di circolarità fra giustizia, carità e verità ${ }^{29}$, dove il rispetto autentico per la verità si propone come l'antidoto alla tentazione di «contrapporre la giustizia alla carità» ${ }^{30}$, idea ribadita nella allocuzione successiva, quella del 22 gennaio $2011^{31}$.

Anche nell'allocuzione alla Rota del 21 gennaio 2012, dedicata alla interpretazione della legge, il Papa, rifuggendo dagli estremi del positivismo e di una arbitraria creatività, invita a cogliere l'intrinseco contenuto giuridico

\footnotetext{
AAS 96 (2004) 351.

AAS 97 (2005) 164.

${ }^{26}$ Ibid., 165.

AAS 98 (2006) 136 e 137.

AAS 99 (2007) 87.

Cfr. AAS 102 (2010) 110-114.

${ }^{30}$ Ibid., 110.

${ }^{31}$ Cfr. AAS 103 (2011) 108 e 113.
} 
della realtà, parlando di «verità giuridica da amare, da cercare e da servire» e di «un senso di vera riverenza nei riguardi della verità sul diritto» ${ }^{32}$.

L'ultima allocuzione alla Rota di Benedetto XVI, quella del 26 gennaio 2013, dedicata al tema del rilievo nella fede sulle intenzioni e capacità matrimoniali, nonché sulle ricadute dell'eventuale mancanza di fede sull'ordinazione del matrimonio al bene dei coniugi, si chiude con una invocazione a favore di coloro che, nella Chiesa, si adoperano «per la salvaguardia della verità e della giustizia riguardo al vincolo sacro del matrimonio» ${ }^{33}$.

Anche papa Francesco non ha mancato di evocare il tema della verità nei suoi discorsi alla Rota Romana. Nella sua prima allocuzione, ossia quella del 24 gennaio 2014, ha infatti collocato proprio nel «servizio alla verità e alla giustizia» l'armonizzazione fra la dimensione giuridica e quella pastorale: «La dimensione giuridica e la dimensione pastorale del ministero ecclesiale non sono in contrapposizione, perché entrambe concorrono alla realizzazione delle finalità e dell'unità di azione proprie della Chiesa ${ }^{34}$. Tracciando poi un profilo ideale del giudice canonico, il Papa mette in luce, oltre all'aspetto umano e pastorale, anche quello propriamente giuridico, dove oltre alla perizia e alla obiettività egli è caratterizzato dal rispetto per la verità. Ma ascoltiamo le sue parole: «Oltre ai requisiti di dottrina giuridica e teologica, nell'esercizio del suo ministero il giudice si caratterizza per la perizia nel diritto, l'obiettività di giudizio e l'equità, giudicando con imperturbabile e imparziale equidistanza. Inoltre nella sua attività è guidato dall'intento di tutelare la verità, nel rispetto della legge, senza tralasciare la delicatezza e umanità proprie del pastore di anime» ${ }^{35}$.

Nella allocuzione del 23 gennaio 2015, trattando del possibile influsso della mentalità definita mondana sulla impostazione del matrimonio, il Papa vede la realizzazione dell'orientamento del diritto alla salus animarum proprio nell'aiutare «a stabilire la verità nel momento consensuale» ${ }^{36}$, qui intesa soprattutto come consonanza alla concezione cristiana dal matrimonio.

Inteso almeno in parte in questa accezione, il tema della verità ritorna in modo prepotente nell'allocuzione del 22 gennaio 2016, dove significativamente la Rota Romana viene definita come il «Tribunale della verità del vin-

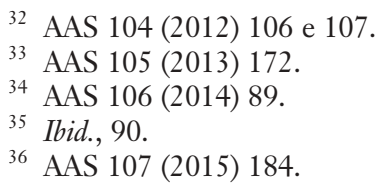


colo sacro». In particolare il Papa, con riferimento alla visione ecclesiale del matrimonio, afferma che la Chiesa, pur misericordiosa verso le situazioni imperfette, deve «insieme proclamare l'irrinunciabile verità del matrimonio secondo il disegno di Dio». Passando poi al tema specifico dell'esercizio della funzione giudiziale, afferma che essa «assiste e promuove l'opus veritatis» ${ }^{37}$, per mezzo del quale la Chiesa riconosce e giudica la verità di un matrimonio concreto.

2.3. Anche nella recente riforma processuale canonica non si è inteso venir meno alla ricerca e alla tutela della verità.

Nel motu proprio Mitis Iudex Dominus Iesus ${ }^{38}$ il richiamo alla verità è assolutamente presente. Già nell'esordio del proemio si afferma infatti che l'evoluzione dell'ordinamento sistematico delle nullità matrimoniali e dello strumento processuale avvennero «ita ut ecclesiastica disciplina magis magisque cum veritate fidei, quam profitebatur, cobaereret»: una verità, dunque, anzitutto in senso dottrinale, finalizzata all'ottenimento della salvezza delle anime: una verità, dunque, anche salvifica.

Il proemio, tornando a riferirsi alla verità nella sua accezione dottrinale, precisa che la revisione processuale approvata ha posto ogni cura affinché fosse «in tuto utique posito principio vinculi matrimonialis indissolubilitatis»; e che la sua conservata collocazione all'interno della dimensione propriamente giudiziale è dovuta «non eo quod rei natura id imponat, sed potius postulatio urgeat veritatis sacri vinculi quammaxime tuendae: quod sane praestant ordinis iudiciarii cautiones», dove emerge con chiarezza che la verità cui qui si fa riferimento sia da intendersi piuttosto in senso storico, ossia come la verità fattuale di ogni singolo matrimonio sottoposto al vaglio ecclesiale della verifica della sua validità.

Se si esaminano poi quelli che MIDI chiama i fundamentalia criteria che stanno alla sua base, non si può non sottolineare che nel primo di essi -nel quale si sancisce la fine dell'obbligo della doppia sentenza conforme- si faccia riferimento al concetto cardine della certezza morale: «sufficere certitudinem moralem a primo iudice ad normam iuris adeptam», concetto che richiede un gra-

37 http://w2.vatican.va/content/francesco/it/speeches/2016/january/documents/papa-francesco_20160122_anno-giudiziario-rota-romana.html.

${ }^{38}$ D'ora in poi MIDI: per comodità di farà costante riferimento al testo normativo per la Chiesa latina. 
do assai alto di verità nella ricostruzione storica e nella possibilità di univoca qualificazione giuridica dei fatti.

Anche il secondo principio - che trova poi una sua applicazione normativa nel nuovo can. $1673 \$ \$ 3-5-$ accompagna l'ampliamento della possibilità del ricorso al giudice unico con l'invito «ne cuilibet laxismo indulgeatur». E, in effetti, il ribadire la preferenza per il collegio di giudici in primo grado e, anzi, la sua necessità ad validitatem in secondo appare chiaramente dettato dalla maggiore garanzia che il giudice collegiale offre di una pronuncia maggiormente aderente alla verità soprattutto storica, meno esposta alle unilateralità di un giudizio monocratico.

Anche nel principio quarto, dove si annuncia la creazione del processus brevior, si nota il rispetto per la verità sia storica sia dottrinale: sia legando la procedibilità di tale forma processuale alla qualità manifesta o evidente della nullità dedotta (cfr. la condizione chiaramente espressa dal nuovo can. 1683, $2^{\circ}$ ); sia affidando al vescovo (diocesano) la decisione, affiché il possibile rischio che pur si accetta di correre a detrimento della indissolubilità del matrimonio venga bilanciato dalla garanzia della autorità di colui «qui in fide et disciplina unitati catholicae cum Petro ob suum pastoris munus quam qui maxime cavet».

Passando all'articolato normativo di MIDI, oltre a quanto già anticipato in merito alla composizione collegiale del tribunale e a quanto si dirà più avanti in merito al valore probatorio delle dichiarazioni delle parti, si deve rilevare che non appare per nulla ridimensionato il ruolo del difensore del vincolo, cui pure spetta il diritto di appello (cfr. can. $1680 \$ 1$ ): anzi, il suo ruolo -col venire meno del controllo obbligatorio reso necessario in vista dell'ottenimento della doppia conforme- acquista una nuova responsabilità, nella pur ragionevole tutela della verità sia storica sia dottrinale da parte della giurisprudenza ecclesiastica.

La ricerca di una verità storica, fattuale, particolarmente accurata appare chiaramente espressa nei requisiti che debbono caratterizzare il libello proposto per il processo breve: esso deve indicare $\ll 1^{\circ}$ facta $[. ..] 2^{\circ}$ probationes [...] $3^{\circ}$ documenta [...]», tutti concetti che fanno indiscutibile riferimento a un oggettivo ancoraggio storico della domanda al reale. Così come la possibilità che il vescovo rimetta la causa all'esame ordinario in caso di non raggiungimento da parte sua della certezza morale (cfr. can. $1687 \$ 1$ ) -ovviamente per un approfondimento istruttorio e di discussione-indica chiaramente che le ragioni della verità prevalgono rispetto alla pur importante celerità, nonché all'esau- 
dimento della domanda dei coniugi, nel processo breve entrambi favorevoli alla dichiarazione della nullità del loro matrimonio.

Infine, il ribadire nel rinnovato can. $1691 \$ 3$ che le cause di nullità del matrimonio sono cause «ad bonum publicum spectantes» significa ribadire che il loro oggetto sfugge alla libera disponibilità delle parti e dello stesso giudice, i quali devono riconoscere la situazione oggettiva che sta alla base della condizione giuridica dedotta in giudizio.

Quanto infine alla Ratio procedendi annessa al MIDI (RP), essa esordisce con una limpida riaffermazione della convinzione che «tribunalium opus respondere valeat fidelibus veritatem declarari postulantibus de exsistentia annon vinculi sui collapsi matrimonii»: la dichiarazione della verità è lo scopo del giudizio canonico. Tale convinzione trova la sua concretizzazione massima nell'art. 12 della RP, nel quale viene ribadita sia la necessità sia la definizione della certezza morale, necessaria a dichiarare la nullità di un patto nuziale. La necessità della certezza morale si propone come una garanzia molto forte della corrispondenza del giudizio canonico alla realtà dei fatti: solo tale corrispondenza, infatti, può assicurare il vero valore pastorale della pronuncia giudiziaria e delle altre scelte che ne conseguono, ad esempio l'ammissione a un nuovo matrimonio.

In conclusione, possiamo affermare che anche in MIDI possiamo reperire le diverse accezioni del concetto di verità che abbiamo individuato nella prima parte delle nostre riflessioni: la verità storica, giuridicamente qualificabile in modo non difforme dalla verità dottrinale, solo sulla base delle quali è possibile accedere a una verità salvifica, veramente corrispondente alla sua natura pastorale.

2.4. L'orientamento alla ricerca della verità appare -anche in una prospettiva comparativa- una caratteristica molto spiccata del modello processuale canonico.

Il processo non è solo funzionale a garantire le regole della contrapposizione fra le parti, che perseguono in esso i propri interessi individuali; nemmeno può essere inteso solo come strumento per la risoluzione del conflitti e per assicurare la certezza dei rapporti giuridici, potendo accontentarsi anche della sola cosiddetta verità processuale; nemmeno può essere visto come funzionale alla attuazione di un progetto politico, per quanto con l'attenzione alla corrispondenza ai fatti; bensì deve essere visto soprattutto come uno strumento di ricerca della verità oggettiva, sulla base della quale 
realizzare la finalità salvifica della vita ecclesiale, anche nella sua dimensione istituzionale ${ }^{39}$.

Per meglio illustrare quanto intendo dire, vorrei sviluppare una considerazione che parte dalla esperienza dalla amministrazione della giustizia penale canonica. In un sistema come quello canonico, non avrebbe un senso (né etico né giuridico) un istituto quale il cosiddetto patteggiamento, ossia l'applicazione concordata di una pena, dove il sistema giudiziario risparmia attività e costi, mentre l'imputato esce dal meccanismo del processo senza ammettere la colpa e accettando una pena minore di quella edittale. Tutto ciò, infatti, avviene sulla base della aprioristica rinuncia a fare verità sia sui fatti, sia sulla effettiva responsabilità dell'imputato e sul grado della stessa.

\section{LO STRUMENTO PROCESSUALE IN VISTA DEL RINOSCIMENTO DELLA VERITÀ}

3.1. Venendo infine ad alcune considerazioni circa il servizio alla verità nel preciso contesto dello strumento processuale, penso importante anzitutto recuperare il giusto significato, al suo interno, del principio del contraddittorio, un criterio che la cultura giuridica anche secolare apprezza come qualificante l'attuarsi di un giusto processo.

Talvolta ho sentito presentare in termini banalizzati la qualità contenziosa del processo matrimoniale, sostenendo che in esso una parte non pretende nulla dall'altra, con la contestuale affrettata messa in discussione della forma processuale quale strumento per l'accertamento della verità dello stato di vita dei fedeli. Certamente, il processo di nullità matrimoniale, pur modellato sul processo contenzioso ordinario, è un processo speciale di natura pubblicistica, essendo interesse della collettività ecclesiale acquisire la certezza in merito: ciò al punto che tale processo non obbedisce al mero principio dispositivo nella produzione delle prove, ma prevede l'iniziativa del giudice nel supplire l'eventuale negligenza delle parti e in vista di evitare una sentenza ingiusta (cfr. can. 1452 e art. 71 DC). Tuttavia, ciò non deve portare a comprimere eccessivamente il diritto delle parti di apportare nel giudizio tutti gli elementi che

${ }^{39}$ Cfr., per una analisi più precisa, ampia e rigorosa dei modelli processuali dei sistemi di common e civil law, del cosiddetto diritto socialista e di quello canonico, il primo capitolo del volume: E. Di BERNARDO, Modelli processuali e diritto probatorio civile, Lateran University Press, Città del Vaticano 2016, 17-83. 
possano essere ritenuti leciti ed utili per dimostrare il loro punto di vista (cfr. can. $1527 \$ 1$ e art. $157 \$ 1 \mathrm{DC}$ ). Anzi, esse devono essere trattate con parità ed equilibrio, senza atteggiamenti paternalistici e autoritari da parte dell'autorità ecclesiale ${ }^{40}$. La normativa canonica riconosce al fedele il diritto a un processo non solo giusto nel merito, ma anche nel rito (cfr. il can. $221 \$ \$ 1-2$ ) e in modo veramente efficace il principio del contraddittorio (e dunque anche la retta contenziosità del processo) è stato descritto nei termini seguenti: «[...] lo scopo del processo è la dichiarazione della verità da parte di un terzo imparziale, dopo che è stata offerta alle parti pari opportunità di addurre argomentazioni e prove entro un adeguato spazio di discussione. Questo scambio di pareri è normalmente necessario, affinché il giudice possa conoscere la verità e, di conseguenza, decidere la causa secondo giustizia» ${ }^{41}$.

Il contraddittorio, quindi, è uno strumento di ricerca e servizio alla verità e alla giustizia.

3.2. Potrebbe sembrare paradossale, ma un secondo principio processuale che appare congruo al riconoscimento e, quindi, al servizio della verità è quello rappresentato dalla presunzione del can. 1060, basata sul fatto incontestabile della celebrazione nuziale e sulla presupposizione che essa sia stata affrontata lealmente dai suoi protagonisti ${ }^{42}$. Una presunzione, dunque, sia che si basa su una visione realistica; sia che esprime un'autentica visione personalistica, in quanto ritiene le persone capaci e responsabili dei loro atti nonché guidate da rette intenzioni nel loro agire.

In particolare, è importante evidenziare l'erroneità della contrapposizione fra favor matrimonii e favor personae (o libertatis, che dir si voglia). Essi trovano il loro equilibrio solo nel riconoscimento della verità, per cui è il favor veritatis che si propone come il vero cardine dell'attività processuale canonica, come la sua chiave di volta. Infatti, non si può pretendere di promuovere la persona e la sua autentica libertà se non nella verità: la tutela dell'istituzione

${ }^{40}$ In questo senso va letta la limitata possibilità di azione del promotore di giustizia (cfr. anche il rinnovato can. $1674 \$ 1,2^{\circ}$ ) e la necessità della istanza di una delle parti per la modifica del dubbio di causa (cfr. il can. 1514 e l'art. 136 DC).

41 BenedeTto XVI, Allocuzione alla Rota Romana del 28 gennaio 2006, AAS 98 (2006) 136.

${ }^{42}$ Cfr. in merito A. S. SÁNCHEZ GIL, La presunzione nella vigente normativa canonica: osservazioni critiche, Ius Ecclesiae 25 (2013) 55-76 e A. S. SÁNCHEZ GIL, La presunzione nella vigente normativa canonica: osservazioni critiche, in AA.Vv., L'istruttoria nel processo di nullità matrimoniale, Libreria Editrice Vaticana, Città del Vaticano 2014, 139-160. 
matrimoniale e la promozione della persona non possono smarrire il loro «riferimento teleologico alla verità ${ }^{43}$, senza il quale tutta la struttura del processo si tramuterebbe in una sequenza di formalismi privi di vita.

La pretesa promozione della persona a scapito della verità -oppure coltivando una sorta di sistema della doppia verità, quasi che si possano separare radicalmente il profilo istituzionale da quello personale- appare funzionale a una sorta di eudemonismo individualistico. Il bene autentico della persona è quello di conoscere la verità del proprio stato di vita ed essere aiutata a osservare le responsabilità che ne derivano, anche se non sempre facili e anzi talvolta gravose.

3.3. Connesso a tale tema è quello del concetto di certezza morale, inteso come cardine dell'ordinamento processuale canonico ${ }^{44}$. La necessità della certezza morale e la sua definizione tecnica sono state ribadite nell'articolo 12 della $\mathrm{RP}$ e, come già più sopra rilevato, l'affermata necessità di questo tipo di certezza presuppone e richiede un alto grado di sicurezza circa il fatto che una eventuale sentenza (affermativa) in tema di nullità matrimoniale corrisponda alla verità oggettiva delle cose.

Tale scelta normativa toglie ogni equivoco in merito alla finalità di mera semplificazione processuale della riforma promossa da papa Francesco e delegittima sue interpretazioni che tendessero a far scivolare le pronunce canoniche in merito sul piano di una implicita modifica del diritto sostanziale o dell'introduzione nel sistema canonico di pronunce costitutive di scioglimento del vincolo, sia da parte dei tribunali sia da parte dei vescovi nel processus brevior.

3.4. Uno strumento utile a pervenire il più possibile alla conoscenza della verità oggettiva e non solo alla costruzione di una verità puramente processuale consiste nel rilievo probatorio che l'ordinamento canonico riconosce alle dichiarazioni delle parti.

Molto è stato scritto in merito -sia su un piano propriamente scientifico, sia talvolta indulgendo a semplificazioni piuttosto sloganistiche- e non è que-

43 Così si esprimeva san Giovanni Paolo II nella sua Allocuzione alla Rota Roma del 29 gennaio 2004, AAS 96 (2004) 348-352 (le parole citate a p. 351).

${ }^{44}$ Cfr. in questo senso e fra i molti possibili contributi quello autorevole di Z. GROCHOLEWSKI, $L a$ certezza morale come chiave di lettura delle norme processuali, Ius Ecclesiae 9 (1997) 417-450. 
sta la sede per riprendere tale dibattito. Circa tale questione vorrei solo suggerire alcune puntualizzazioni.

Anzitutto che si tratta propriamente delle dichiarazioni giudiziali delle parti, ossia quelle rese in giudizio pro se e contra vinculum e che la DC autorizza a chiamare -come già nella comune prassi dei tribunali- confessioni giudiziali (cfr. art. $179 \$ 2$ ). Non si deve dimenticare ciò nell'attribuire valore probatorio a tali dichiarazioni in cause ove si tratta di diritti per sé indisponibili alle parti.

In secondo luogo che -per quanto l'affermazione del nuovo can. $1678 \$ 1$ sia volta in positivo- una analisi credo pacata delle condizioni previste circa la possibilità che esse acquisiscano valore di prova piena porta a concludere che la relativa disciplina non è mutata di molto. Infatti, se si confronta la nuova norma del can. $1678 \$ 1$ con la disciplina in precedenza disegnata dalla lettura combinata dei cann. $1536 \$ 2$ e 1679, si può constatare quanto segue. In entrambi i casi l'effetto probatorio pieno è solo eventuale e la sua valutazione è affidata al libero apprezzamento del giudice ${ }^{45}$, mentre si richiedono; a) eventuali testimonianze di credibilità; b) la valutazione di indizi e circostanze di contorno (adminicula); c) l'assenza di elementi oggettivi contrari, che consentano il conseguimento della certezza morale, espressa nel can. $1536 \$ 2$ (tutt'ora vigente) dal concetto di conferma omnino di tali dichiarazioni da parte degli altri elementi di prova presenti in atti.

Da quanto appena detto, in terzo luogo, discende che nel valutare le dichiarazioni o confessioni giudiziali delle parti occorrerà anche distinguere attentamente la sincerità soggettiva (ossia la volontà di dire il vero) dalla oggettività, ossia la capacità di dire il vero che andrà commisurata dal raffronto di quelle dichiarazioni con gli elementi storici presenti in atti e idonei appunto a confermarle o a confutarle.

Quello che andrà evitato -come già anche in dottrina e in giurisprudenza si è più volte osservato- è il porsi nella prospettiva di una sorta di autocertificazione della nullità matrimoniale e della riduzione del processo a una dinamica di solo foro interno. In una sentenza di qualche anno fa ${ }^{46}$ si ricordava il pressante monito di papa Innocenzo III: «cum nimis indignum sit, iuxta legitimas sanctiones, ut quod sua quisque voce dilucide protestatus est, in eundem casum

${ }^{45}$ Non si tratta cioè di una specie di prova legale, che creerebbe una sorta di nuovo formalismo: la dichiarazione della parte esaurisce la ricerca probatoria.

${ }^{46}$ La coram Davino 2 agosto 1993, in RRDec., vol. LXXXV, 616-617. 
proprio valeat testimonio infirmare». Ossia che taluno, con le sue sole parole, possa porre nel nulla impegni di rilievo pubblico da lui in precedenza solennemente assunti.

Infine, la spia della serietà con la quale questa possibilità probatoria venga usata sarà anche il verificare con quale disponibilità si presterà fede alle dichiarazioni delle parti contrarie al riconoscimento della nullità del patto nuziale. Ad esempio, siccome il convenuto che si oppone alla domanda è a sua volta tenuto a provare $i$ fatti che intende addurre a fondamento della sua tesi ${ }^{47}$, anche le sue dichiarazioni -se avallate da referenze di credibilità, se accreditate da elementi oggettivi, se non confutate da elementi contrari- dovranno essere accolte con la medesima disponibilità a riconoscere loro peso probatorio.

3.5. Vorrei concludere col riferire una esperienza personale. Negli ultimi tempi, mi è capitato di imbattermi -nelle difese di cause di nullità matrimoniale dal malcerto fondamento, se non addirittura chiaramente infondatenell'insistito appello alla misericordia dei giudici, come pure all'allegazione di scritti di sacerdoti che invocano misericordia per persone che raccomandano all'attenzione del tribunale. Non mi sembra una modalità corretta di argomentazione, che anzi potrebbe portare a una banalizzazione, a un uso indebito e strumentale della grande idea della misericordia.

In merito, vorrei sottoporre alla libera e critica valutazione, quanto ho inserito in una sentenza, proprio a commento degli scritti di due sacerdoti che facevano leva su tale tipo di argomento:

«nei loro scritti $i$ due sacerdoti fanno abbondante ricorso al tema della misericordia e tale invito lo si comprende perfettamente nel tipo di testo da loro prodotto e anzi lo si apprezza cordialmente come espressione di sensibilità pastorale degli scriventi. Tuttavia, un concetto così serio e importante come quello della misericordia -proprio perché esso non venga svilito [...]- deve essere proporzionato al contesto in cui ci si trova e alle esigenze della verità.

A questo proposito, va ricordato che nel varare la recente riforma processuale lo stesso papa Francesco ba ricordato la natura solo dichiarativa del processo

47 Non si intende dire che il convenuto debba provare la validità del matrimonio, perché questa è presupposta dall'ordinamento; ma che egli deve provare la verità dei fatti storici che adduce come contrari alla tesi che la parte attrice è onerata di dimostrare. 


\section{PAOLO BIANCHI}

di nullità matrimoniale, che deve quindi riconoscere come nulli solo $i$ matrimoni che siano tali e che tali siano provati con certezza morale (cfr. art. $12 \mathrm{RP}$ ). Il giudice, in altre parole, non ba la disponibilità sulla materia del vincolo coniugale; per questo egli non ba il potere di dichiarare nullo -per misericordia-un matrimonio che non è provato lo sia, magari per andare incontro al sincero desiderio di ricevere l'Eucaristia di una persona che ba delle indubbie benemerenze ecclesiali, [...].

Il giudice esercita la misericordia -nei limiti della sua funzione istituzionale: ma ogni atto di misericordia umana è limitato, essendo solo quella di Dio la misericordia perfetta, che sa integrare in sé pienamente anche la giustiziacercando di svolgere bene il suo umile compito di mettere al servizio delle persone lo strumento prescelto dalla Chiesa per il discernimento ufficiale circa il loro stato di vita, dando però loro una risposta corrispondente alla verità che si è potuta ricostruire.

L'ammissione all'Eucaristia di persone che vivono in condizioni oggettivamente irregolari (nel caso, a valle di un matrimonio valido) è una valutazione pastorale di altro tipo, ulteriore all'accertamento giudiziale dello stato di vita delle persone, che non può che essere fatto nella verità. $\grave{E}$ quella valutazione cui fa cenno don [...] nel suo scritto e della quale tratta l'esortazione apostolica Amoris laetitia nel suo capitolo VIII e nella quale l'interessato potrà farsi guidare da persone idonee ad assisterlo sotto questo profilo». 


\section{Bibliografía}

BenedetTo XVI, Allocutio Ad Tribunal Rotae Romanae (28 gennaio 2006), AAS 98 (2006) 135-138.

-, Allocutio Ad Tribunal Rotae Romanae in inauguratione Anni Iudicialis (28 gennaio 2007), AAS 99 (2007) 86-91.

—, Allocutio Ad Sodales Tribunalis Romanae Rotae (29 gennaio 2010), AAS 102 (2010) 110-114.

—, Allocutio Ad Sodales Tribunalis Rotae Romanae (22 gennaio 2011), AAS 103 (2011) 108-113.

-, Allocutio Ad Sacrae Rotae Romanae Tribunal, Occasione Inaugurationis Anni Iudicialis (21 gennaio 2012), AAS 104 (2012) 103-107.

—, Allocutio Ad Romanae Rotae Tribunal (26 gennaio 2013), AAS 105 (2013) 168-172.

Di Bernardo, E., Modelli processuali e diritto probatorio civile, Lateran University Press, Città del Vaticano 2016, 17-83.

ERLebach, G. (a cura di), Le allocuzioni dei Sommi Pontefici alla Rota Romana, Libreria Editrice Vaticana, Città del Vaticano 2004, 24, n. 12.

Francesco I (PAPA), Allocutio Ad Omnes Participes Tribunalis Romanae Rotae (24 gennaio 2014), AAS 106 (2014) 89-90.

-, Allocutio Ad Sodales Tribunalis Romanae Rotae (23 gennaio 2015), AAS 107 (2015) 182-185.

-, Discorso del Santo Padre Francesco in occasione dell'inaugurazione dell'anno giudiziario del Tribunale della Rota Romana (22 gennaio 2016) (http://w2.vatican.va/content/francesco/it/speeches/2016/january/documents/papa-francesco_20160122_anno-giudiziario-rota-romana.html).

Giovanni Paolo II (SAN), Allocutio Ad Decanum Sacrae Romanae Rotae ad eiusdemque Tribunalis Praelatos Auditores, ineunte anno iudiciali (17 febbraio 1979), AAS 71 (1979) 422-427.

-, Allocutio Ad Tribunalis Sacrae Romanae Rotae Decanum, Praelatos Auditores, Officiales et Advocatos, novo Litibus iudicandis ineunte anno: de veritate iustitiae matre (4 febbraio 1980), AAS 72 (1980) 172-178.

-, Allocutio Ad Praelatos Auditores ceterosque Officiales et Administros Tribunalis Sacrae Romanae Rotae: in ferendis sententiis responsis Dicasteriorum et Tribunalium Sedis Apostolicae standum est (24 gennaio 1981), AAS 73 (1981) 228-234.

-, Allocutio Ad Rotae romanae auditores coram admissos (5 febbraio 1987), AAS 79 (1987) 1453-1459. 
- Allocutio Ad Excmos. Legatos et Oratores apud Sedem Apostolicam permanenter Missos (9 gennaio 1988), AAS 80 (1988) 1133-1143.

-, Allocutio Ad Romanae Rotae Praelatos, Auditores, Officiales et Advocatos Anno Iudiciali Ineunte (18 gennaio 1990), AAS 82 (1990) 872-877.

-, Allocutio Ad Romanae Rotae Auditores coram Admissos (29 gennaio 1993), AAS 85 (1993) 1256-1260.

-, Allocutio Ad Romanae Rotae Iudices et Administros coram Admissos (28 gennaio 1994), AAS 86 (1994) 947-952.

-, Allocutio Ad Romanae Rotae Praelatos Auditores (17 gennaio 1998), AAS 90 (1998) 781-785.

-, Allocutio Ad Romanae Rotae praelatos Auditores (21 gennaio 1999), AAS 91 (1999) 622-627.

—, Allocutio Ad Romanae Rotae Tribunal (28 gennaio 2002), AAS 94 (2002) 340346.

-, Allocutio Ad Rotam Romanam Habita (29 gennaio 2004), AAS 96 (2004) 348-352.

-, Allocutio Ad Tribunal Rotae Romanae Iudiciali Ineunte Anno (29 gennaio 2005), AAS 97 (2005) 164-166.

Giovanni XXIII (SAN), Allocutio Ad Praelatos Auditores cetreoque Officiales, Advocatos et Procuratores Tribunali Sacrae Romanae Rotae (13 dicembre 1961), AAS 53 (1961) 817-820.

GROCHOLEWSKI, Z., La certezza morale come chiave di lettura delle norme processuali, Ius Ecclesiae 9 (1997) 417-450.

Paolo VI (Beato), Allocutio Ad Tribunalis Sacrae Romanae Rotae Decanum, Praelatos Auditores, Officiales et Advocatos, ineunte anno iudiciali (28 gennaio 1978), AAS 70 (1978) 181-186.

PIO XII, Allocutio Ad Praelatos Auditores ceterosque Officiales et Administros Tribunalis S. Romanae Rotae necnon eiusdem Tribunalis Advocatos et Procuratores (2 ottobre 1940), AAS 34 (1942) 338-343.

-, Allocutio Ad Praelatos Auditores ceterosque Officiales et Administros Tribunalis S. Romanae Rotae necnon eiusdem Tribunalis Advocatos et Procuratores (2 ottobre 1944), AAS 36 (1944) 281-290.

SÁNCHEZ GIL, A. S., La presunzione nella vigente normativa canonica: osservazioni critiche, Ius Ecclesiae 25 (2013) 55-76.

- La presunzione nella vigente normativa canonica: osservazioni critiche, in AA.VV., L'istruttoria nel processo di nullità matrimoniale, Libreria Editrice Vaticana, Città del Vaticano 2014, 139-160. 